\title{
Autosomal recessive optic atrophy, OPA7 type
}

INSERM

\section{Source}

INSERM. (1999). Orphanet: an online rare disease and orphan drug data base. Autosomal recessive optic atrophy, OPA7 type. ORPHA:227976

A rare, syndromic, hereditary optic neuropathy disorder characterized by early-onset, severe, progressive visual impairment, optic disc pallor and central scotoma, variably associated with dyschromatopsia, auditory neuropathy (e.g. mild progressive sensorineural hearing loss), sensorimotor axonal neuropathy and, occasionally, moderate hypertrophic cardiomyopathy. 